\title{
DETERMINACIÓN SEROLÓGICA DE LA INFECCIÓN CON Mycoplasma hyopneumoniae EN UNA GRANJA PORCINA DE LIMA
}

\author{
Marlon Torres A. ${ }^{1}$, Sonia Calle E. ${ }^{2,3}$, Hermelinda Rivera G. ${ }^{2}$, Carlos Camacho S. ${ }^{4}$, \\ Néstor Falcón P. ${ }^{5}$ y César Alzamora P. ${ }^{1}$
}

\section{Abstract}

\begin{abstract}
A serological study was conducted to identify the age of highest infection with Mycoplasma hyopneumoniae in pigs under an intensive rearing system in the Lima province, during the period April - August, 2002. Blood samples were collected fortnightly from birth till 16 months of age in 30 piglets. The commercial test ELISA Chekit ${ }^{\circledR}$-HyoptestII was used for the detection of antibodies. The 66.3\% (20/30) of the animals showed antibodies against $M$. hyopneumoniae. The critical period for starting the infection occurred at 9-10 weeks of age. Sex and number of parturition did not affect the time of initial infection.
\end{abstract}

Key words: swine, Mycoplasma hyopneumoniae, antibodies, ELISA

\section{Resumen}

Se realizó un estudio serológico para determinar el momento de mayor infección con Mycoplasma hyopneumoniae de una granja porcina de crianza intensiva en la provincia de Lima, en el periodo abril-agosto del 2002. Se tomaron muestras quincenales de sangre en 30 animales desde el nacimiento hasta las 16 semanas de edad. Se utilizó la prueba comercial de ELISA Chekit ${ }^{\circledR}$-Hyoptest-II para la detección de anticuerpos. El 66.3\% (20/ 30) de los animales presentaron anticuerpos contra M. hyopeumoniae determinándose que el mayor porcentaje seroconvirtió a las 12 semanas, por lo que se concluye que el periodo crítico corresponde a las 9-10 semanas de edad donde el M. hyopneumoniae inicia la infección que condujo a la seroconversión. El sexo y número de partos de la madre no afectó el momento de la infección.

Palabras clave: porcino, Mycoplasma hyopneumoniae, anticuerpo, ELISA

\author{
${ }^{1}$ Práctica privada \\ ${ }^{2}$ Laboratorio de Microbiología y Parasitología Veterinaria, FMV-UNMSM \\ ${ }^{3}$ E-mail: calleson@gmail.com \\ ${ }^{4}$ Laboratorios Bang \\ ${ }^{5}$ Laboratorio de Medicina Veterinaria Preventiva, FMV-UNMSM
}




\section{INTRODUCCIÓN}

El Mycoplasma hyopneumoniae es reconocido como uno de los más importantes agentes infecciosos que producen problemas respiratorios en porcinos. Es el agente causal de la Neumonía Enzoótica y está considerado como un microorganismo de alta prevalencia y de distribución mundial (Ross, 1986). La sintomatología clínica y las pérdidas económicas son de diversa magnitud y como resultado de una compleja interacción con bacterias secundarias, deficiencias en el manejo y condiciones medio ambientales adversas (Camacho, 2000).

En los últimos años, el $M$. hyopneumoniae ha demostrado ser el patógeno primario más importante del Complejo de la Enfermedad Respiratoria Porcina (Halbur, 1997); así mismo, se reconoce que potencia la neumonía causada por el virus del Síndrome Reproductivo y Respiratorio Porcino (SRRP) (Thacker et al., 1999) y posiblemente interactúa con el Circovirus Tipo 2 en la Neumonía Intersticial (Ohlinger et al., 2002). En el Perú se ha demostrado la presencia del M. hyopneumoniae en gorrinos a la edad de beneficio (Huallanca, 1999); sin embargo, no se han elaborado programas para su control basados en el monitoreo serológico.

El objetivo del presente trabajo fue determinar, mediante serología, el periodo de infección con M. hyopneumoniae en un sistema de producción porcina intensiva típico del Perú, a fin de contribuir con la caracterización de la infección y la elaboración de estrategias de prevención y control de la enfermedad en este sistema de producción.

\section{Materiales y Métodos}

El estudio se realizó entre abril y agosto del 2002 en una granja porcina de crianza intensiva y con producción en un solo sitio, ubicada en el distrito de Puente Piedra, provincia de Lima. Se seleccionaron 15 lecho- nes hembras y 15 machos al nacimiento (Teorema de Límite Central), procedentes de madres multíparas ( 2 a 4 partos) que estaban en buen estado de salud al inicio del ensayo. La granja no vacuna contra Mycoplasma hyopneumoniae y los animales experimentales no recibieron antibióticos micoplasmicidas durante el ensayo. El manejo de los animales fue el rutinario de la granja.

Se extrajeron muestras de sangre, por punción de la vena cava, con intervalos de 2 semanas desde el segundo día de edad hasta las 16 semanas de vida. Las muestras se llevaron allaboratori de Bacteriobgía de la Facultad de M edicina Veterinaria de la Un iversidad $\mathrm{N}$ acional $\mathrm{M}$ ayor de San M arcos para su almacenam énto y posterír procesamiento.

La detección de anticuerpos contra $M$. hyopneumoniae se realizó mediante la prueba comercial de ELISA Chekit ${ }^{\circledR}$-Hyoptest-II, siguiendo las instrucciones del fabricante. $\mathrm{La}$ prueba fue considerada válida al obtener una diferencia en la Densidad Óptica (DO) entre los controles positivos y negativos mayor a 0.3 .

En el análisis de los resultados de laboratorio se utilizó la siguiente fórmula:

$\left(\mathrm{DO}_{\text {muestra }}-\mathrm{DO}_{\text {neg }}\right) /\left(\mathrm{DO}_{\text {pos }}-\mathrm{DO}_{\text {neg }}\right) \times 100 \%$

Los resultados mayores a $30 \%$ se consideraron positivos, los menores al $20 \%$ fueron considerados negativos, y aquellos entre el 20 y el $30 \%$ se consideraron como sospechosos. Este último caso indica que el animal estaría saliendo de o comenzando la infección, por lo que en muestras sospechosas se recurrió a las muestras previas y posteriores para esclarecer su situación.

En el análisis estadístico se utilizó la prueba de Kaplan Meier para determinar la curva de supervivencia. De esta manera se pudo determinar el momento que seroconvirtió el mayor porcentaje de animales. Para evaluar la relación entre el momento de infección con las variables sexo de la cría y número de partos de la madre, se utilizó la prueba de Regresión de Cox con un intervalo de confianza del $95 \%$. 


\section{Resultados}

Todos los animales mostraron anticuerpos contra M. hyopneumoniae desde el nacimiento hasta las cuatro semanas de edad, observándose una disminución sostenida a partir de la sexta hasta la décima semana de edad (Fig. 1). Posteriormente, a las 12 y 16 semanas de edad se llegó a tener más del 30\% de animales serorreactores. Por otro lado, se determinó que el $66.7 \%$ de los animales muestreados (20/30) seroconvirtieron por infección con $M$. hyopneumoniae.

Mediante la prueba de Kaplan Meier se determinó que el mayor porcentaje de lechones infectados seroconvirtieron a las 12 semanas de edad (Fig. 2). Esta prueba demostró que no hay diferencias significativas entre hembras y machos sobre el momento de seroconversión, resultado que fue confirmado con la regresión de Cox $(\mathrm{p}=0.986)$.

La prueba de Kaplan Meier mostró ciertas diferencias en el momento que seroconvirtieron los lechones con relación al número de parto de la madre; sin embargo, la regresión de Cox no llegó a demostrar esta relación.

\section{Discusión}

El factor predisponente del Mycoplasma hyopneumoniae para causar infecciones secundarias y potenciar a otros agentes patógenos respiratorios determina que su control deba ser el primer paso para la prevención de la Enfermedad Respiratoria Porcina.

La seropositividad del total de los lechones desde el nacimiento hasta la cuarta semana de edad (Fig. 1), debida a la transferencia de anticuerpos calostrales de la madre al lechón, demuestra la activa presencia del $M$. hyopneumoniae en la granja, ya que se trata de madres no vacunadas (Clark, 1999). La infección en las marranas posiblemente se encuentra favorecida por el tipo de manejo y el sistema de producción de la granja (Suprenant, 2001).

Los anticuerpos maternales de lechones que son amamantados por madres naturalmente infectadas decrecen gradualmente en un periodo de seis semanas (Clavijo et al., 2002; Thacker, 1997) o menos (Clark, 1999); sin embargo, en este estudio los anticuerpos maternales estuvieron presentes hasta las ocho semanas de edad (Fig. 1).

Las pruebas estadísticas demostraron que la mayor parte de los animales seroconvirtieron a las 12 semanas de edad (Fig. 2). Este resultado, junto con la observación del comportamiento serológico que tuvieron los animales hasta esa edad, indicaría que el periodo crítico para la infección fue entre las 9 y 10 semanas de edad, es decir, al final de la etapa de recría. La variación en la edad de la seroconversión puede deberse a la variación biológica en la fuerza de la respuesta inmune humoral que existe entre individuos con igual estímulo antigénico (Stevenson, 1999), aunque en aquellos que seroconvirtieron tardíamente se pudo deber a que se infectaron en la etapa de acabado.

Al parecer, en el desarrollo de la seroconversión contra $M$. hyopneumoniae es más importante la ausencia de anticuerpos maternales en la mayor parte de la población que sólo en algunos individuos. En un trabajo previo (Calle et al., 2003), se encontró en una población que todos los animales estaban positivos a PCR de M. hyopneumoniae desde las 2 hasta las 16 semanas de edad; en cambio, en el presente trabajo, un $20 \%$ de los lechones no tenían anticuerpos maternales a la sexta semana. Teniendo en cuenta otros estudios (Thacker y Thacker, 1999) donde se demuestra la importancia de esta protección en el momento de la presentación y severidad de la infección, se presumiría que los animales susceptibles al desarrollo de la infección por la ausencia de anticuerpos tendrían que seroconvertir antes; sin embargo los resul- 


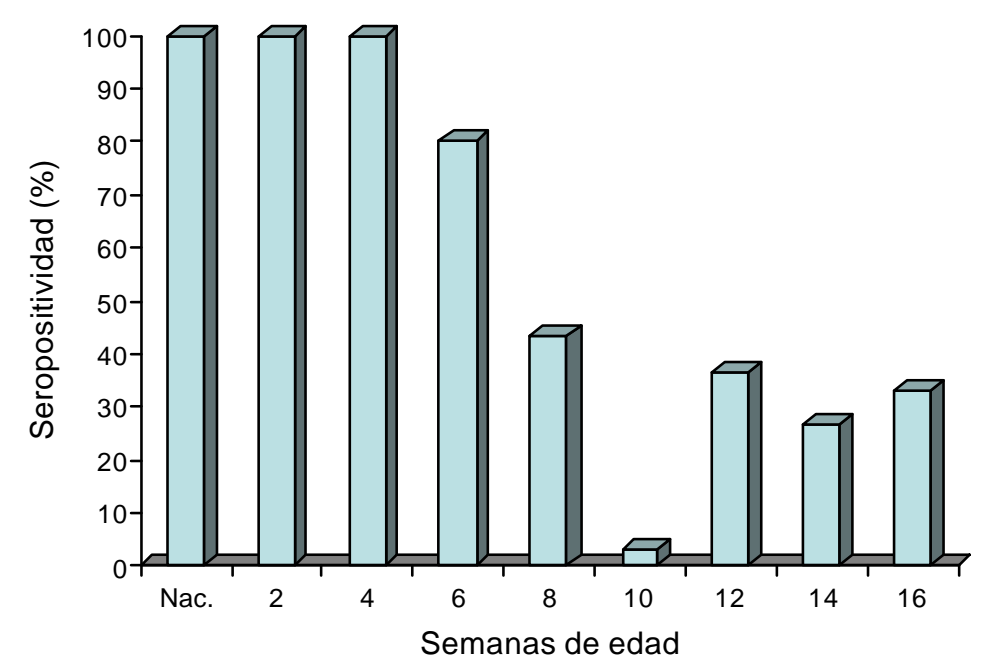

Figura 1. Nivel de seropositividad a Mycoplasma hyopneumoniae (\%) en lechones de una granja de cerdos de crianza intensiva en la zona de Lima, distribuido según la edad de los animales (2002)

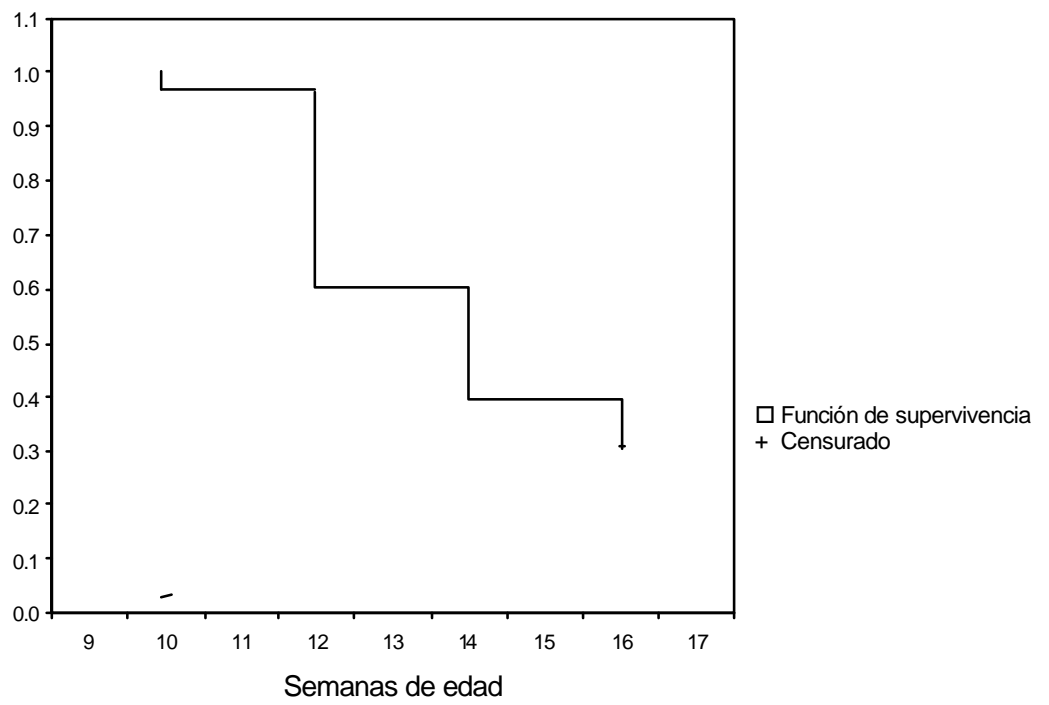

Figura 2. Curva de supervivencia sobre el comportamiento serológico de Mycoplasma hyopneumoniae en lechones de una granja porcina de crianza intensiva en la zona de Lima (2002)

tados indican que la mayoría de los animales seroconvirtieron a partir de las 12 semanas de edad. Se sabe que la inmunidad materna contra $M$. hyopneumoniae previene al individuo del desarrollo de lesiones mientras es expuesto al contagio (Jayappa et al., 2001), y por lo observado en este ensayo, la persis- tencia de la inmunidad en algunos individuos del grupo sería útil para disminuir la presión de la infección en la población. Sin embargo, cuando la mayor parte de los animales del lote dejan de contar con la protección de la inmunidad pasiva, la actividad del $M$. hyopneumoniae prevalece. 
No sólo el estado inmunitario de la población estaría contribuyendo al desarrollo del Micoplasma, sino que algunos factores ambientales podrían estar involucrados (Janke, 1997). En este estudio, el periodo entre las 9 y 10 semanas de edad fue el más crítico y coincidió con los últimos días de permanencia en el área de recría y su traslado al área de engorde. El aumento de desechos orgánicos por animal en el área de recría origina un aumento en la concentración de gases, los cuales son muy difíciles de manejar en ambientes de ventilación natural, creándose situaciones a favorables al Mycoplasma en el tracto respiratorio por la disminución de los mecanismos de defensa (Thacker y Thacker, 1999). Además, el crecimiento de los animales hace que se disminuya el área por animal y por lo tanto el volumen de aire por kilogramo de peso vivo, lo que representa un factor importante en la presentación de problemas respiratorios en porcinos criados en confinamiento. Por otro lado, el traslado a las áreas de engorde, el cambio de ambiente y el reagrupamiento de los animales de acuerdo al tamaño y al sexo son factores de estrés que sumados al estado inmunitario de la población pudieron haber contribuido a una actividad más intensa del Mycoplasma.

El uso de vacunas ayudaría para evitar el "vacío inmunitario" y asegurar la presencia de la inmunidad, tanto celular como humoral. Para determinar el momento de la vacunación es necesario tener en cuenta la presencia de la inmunidad materna al momento de la aplicación, y que en este caso, los animales no tienen que ser negativos a los anticuerpos maternales para que la vacunación sea exitosa contrariamente a lo que suceden con otras vacunas (Thacker y Thacker, 2001). No obstante, es posible que niveles elevados de anticuerpos maternos al momento de la vacunación puedan ser contradictorios para la respuesta inmunitaria (Thacker et al., 1998; Jayappa et al., 2001; Carranza et al., 2002). De acuerdo a este estudio, se podría decir que el momento adecuado para la aplicación de una vacuna contra $M$. hyopneumoniae en la granja del estudio sería entre las siete y las ocho semanas de edad.
Se tiene evidencia de una mayor prevalencia de neumonía y pleuritis en machos castrados que en hembras (Christensen et al., 2000); sin embargo, esto no pudo ser probado en este estudio ya que se trabajaron con machos enteros, y porque la crianza fue mixta durante la etapa de recría.

\section{Conclusiones}

- El $66.3 \%$ de los lechones fueron seroreactores a $M$. hyopneumoniae.

- Se consideró las 12 semanas de edad como el periodo crítico para la seroconversión, estimándose que el Micoplasma inicia su actividad entre la $9^{\mathrm{a}}$ y $10^{\mathrm{a}}$ semana de edad.

- El sexo de la cría y el número de parto de la madre no influyeron en el momento de la infección con $M$. hyopneumoniae.

\section{LITERATURA CITADA}

1. Camacho, C. 2000. Principales enfermedades que afectan a la porcicultura peruana. Memorias II Congreso Nacional de Porcicultura. Lima, Perú.

2. Carranza, A.; N. Pereira; A. Ambrogi. 2002. Interferencia de los anticuerpos maternales en la vacunación contra Mycoplasma hyopneumoniae de campo. Memorias I Congresso Latinoamericano de Suinocultura. p 17-18. Foz de Iguazú, Brasil.

3. Calle, S.; M. Torres; M. Cerón; E. Zacarías; N. Falcón; S. Morales. 2003. Evaluación de la cinética de la infección con Mycoplasma hyopneumoniae en porcinos de crianza intensiva. Memorias III Congreso Nacional de Porcicultura. Lima, Perú.

4. Christensen, G.; V. Sorensen; J. Mousing. 2000. Enfermedades del sistema respiratorio. En: Enfermeda- 
des del cerdo. $8^{a}$ ed. Straw, B. (ed). p 913-940. Ed. Intermedica. Buenos Aires.

5. Clark, L.K. 1999. Mycoplasma hyopneumoniae: serology/vaccinology. Proc. American Association of Swine Practitioners Conference. p 339-343. St. Louis, USA.

6. Clavijo, A.M; M. Rolo; A. Sandoval; C. Ramírez; C. Alfaro; L. Peroza; A. Tonelli; M.J. Alonso. 2002. Cinética de anticuerpos maternos e inducidos por una vacuna contra Mycoplasma hyopneumoniae. Memorias I Congreso Latinoamericano de Suinocultura. $\mathrm{p}$ 7-8. Foz de Iguazú, Brasil.

7. Halbur, P. 1997. Making the diagnosis with serology, antigen detection and PCR. Proc. Iowa State University Veterinary Medicine Seminar. p 3-7. Iowa, USA.

8. Huallanca, A. 1999. Determinación de reactores positivos a Mycoplasma hyopneumoniae en cerdos sacrificados en un camal frigorífico. Tesis de Médico Veterinario. Facultad de Medicina Veterinaria, Univ. Nacional Mayor San Marcos. Lima. 32 p.

9. Janke, B. 1997. Pathogenesis of Mycoplasmal pneumonia, samples for diagnosis. Proc. Iowa State University Veterinary Medicine Seminar. p 1-2. Iowa, USA.

10. Jayappa, H.; R. Davis; V. RappGabrielson; T. Wasmoen; E. Thacker; B.J. Thacker. 2001. Evaluation of the efficacy of Mycoplasma hyopneumoniae bacterin following immunization of young pigs in the presence of varying levels of maternal antibodies. Proc. American Association of Swine Veterinarians Annual Meeting. p 237241. Nashville, USA.

11. Ohlinger, V.; K. Pesch; C. Keller. 2002. Four diseases linked to Porcine Circovirus II. Pig International 32: 17-20.
12. Ross, R.F. 1986. Mycoplasmal disease. En: Disease of swine. $6^{\mathrm{a}}$ ed. Cap. 40. A.D. Leman (ed). Iowa State University Press. Ames, USA.

13. Stevenson, G.W. 1999. Common mistakes in interpretation of population serology. Proc. American Association of Swine Practitioners. p 339-343. St. Louis, USA.

14. Surprenant, Ch. 2001. Mycoplasma hyopneumoniae: serologic interpretation of herd profiles. Proc. American Association of Swine Veterinarians Annual Meeting. p 477. Nashville, USA.

15. Thacker, E.L. 1997. Mycoplasma vaccines: What we know, what we don't know. Proc. Iowa State University Veterinary Medicine Seminar. p 11-13. Iowa, USA.

16. Thacker, B.; T. Boettcher; E. Anderson; E. Thacker; T. Young. 1998. The influence of passive immunity of serological responses to Mycoplasma hyopneumoniae vaccination. Proc. $15^{\text {th }}$ International Pig Veterinary Society Congress. Birminghan, United Kingdom.

17. Thacker, E.L; P. Halbur; F. Ross; R. Thanawongnuwech; B. Thacker. 1999. Mycoplasma hyopneumoniae potentiation of porcine reproductive and respiratory syndrome-virus induced pneumonia. J. Clin. Microbiol. 37: 620-627.

18. Thacker, E.L.; B.J. Thacker. 1999. Mycoplasma hyopneumoniae and PRRS in the finisher. Proc. American Association of Swine Practitioners Annual Meeting. p 483-485. St. Louis, USA.

19. Thacker, B.J.; E.L. Thacker. 2001, Influence of maternally-derived antibodies on the efficacy of a Mycoplasma hyopneumoniae bacterin. Proc. American Association of Swine Veterinarians Annual Meeting. p 513516. Nashville, USA. 